\title{
Mindfulness in Early Signs to Manage Software Projects in the Face of Uncertainty
}

\author{
Marcelo Luiz Monteiro Marinho1,2*, Suzana Cândido de Barros Sampaioํㅜ, Alexandre José \\ Henrique de Oliveira Luna ${ }^{3}$, Telma Lúcia de Andrade Lima ${ }^{2}$, Hermano Perrelli de Moura ${ }^{3}$ \\ ${ }^{1}$ Federal Rural University of Pernambuco (UFRPE) Statistics and Informatics Department (DEINFO) Recife, PE, \\ Brazil. \\ 2 Federal Rural University of Pernambuco (UFRPE) Postgraduate Programme in Administration and Rural \\ Development (PADR) Recife, PE, Brazil. \\ ${ }^{3}$ Federal University of Pernambuco (UFPE) Informatics Center (CIn) Recife, PE, Brazil. \\ * Corresponding author. Tel.: +55 081 3320-6491; email: marcelo.marinho@ufrpe.br \\ Manuscript submitted April 5, 2017; accepted May 10, 2017.
}

doi: 10.17706/jsw.12.7.526-545

\begin{abstract}
Context: In recent years, companies have undergone a renewal of management strategies while searching for space in the market. The involvement of these companies in developing innovative software projects as a form of differentiation in products and services is common. Innovative projects are uncertain by nature, since the behaviour and evolution of the market and technological development may not be predicted. There is a natural relation between software projects that have an innovation degree and uncertainties. Objective: The aim of this paper is to discuss some aspects related to uncertainty management to understand the recommendations that positively influence project management in the face of uncertainties and the method of using the knowledge acquired during this study to provide strategic orientations that can be used by the industry to improve organizations' performance and their projects. Method: This study was built on evidence-based software engineering and provides subsidy for the need to address uncertainty in software projects in order to reduce the negative impacts caused by them. In this article, the interviews' results and focus group conducted with project managers and project management researchers are presented. The interviews and focus group aimed to assess the previous concepts as well as the new practical ones. Results: During the interviews, the concept of early signs was designed and associated with the management of software project uncertainties. In addition, project managers became aware of the mindfulness concept and its use for observing projects' uncertainties in order to ensure its success. Based on information collected from managers and literature review, a series of recommendations were drawn. Furthermore, the recommendations were evaluated by a focus group. Finally, a series of recommendations were designed in order to help the software project managers. Conclusion: This study contributes to software project management by developing recommendations to manage uncertainties.
\end{abstract}

Key words:. Software project management, early signs, uncertainty management, uncertainty in software projects.

\section{Introduction}

Current market demands involve innovation, change in production philosophy, and new processes. Due to the great changing power in today's marketplace, companies need to adapt to the real-time philosophy. Regardless of the business sector, software projects are always changing, thus often differentiating them 
from their competitors and allowing them to grow [1], [2].

It is common for innovative software projects to work in turbulent and uncertain places and scenarios, thus requiring management flexibility and the ability to anticipate future problems [3]. In addition, traditional management methods are unable to predict and manage uncertainties efficiently [4].

Risk analysis and software risk management have been popular topics since the 1990s. These subjects have a very important role during planning and project control [5]. However, despite being of fundamental use in innovative software projects, the tools developed for them remain considerably primitive [6].

Dealing with known risks assumes a mechanistic approach. Managers often deal with risks through corrective actions implemented in accordance with the plan to minimize costs and use the reserve budget only when necessary. However, the project manager who only relies on risk management can work under the false impression that all the project's unknowns have been worked out.

Risk management methods do not usually favour rapid analysis or large amounts of information to be analysed. It does not necessarily reveal whether a risk will come true. However, the analysis assigns a seriousness level, its effect on the project, and the likelihood that the risk indeed becomes a reality [7].

The success of risk management measures depend on how and when the identified actions are going to be implemented to combat the negative impact, which in turn depends on how well the uncertainty management strategies are applied. Risk and uncertainty management should therefore be considered complementary approaches.

We believe that risk management is essential as it reduces the overall uncertainty level associated with the project and that there are many well-established risk management techniques that, if properly applied, can help in uncertainty management. We consider that while risk management remains an important strategy, the project manager also needs strategies to manage uncertainty, that is, to deal with areas and aspects that risk management cannot handle.

Thus, uncertainty management becomes essential for risk management as it provides strategies for the manager to more efficiently transform the unknown to known. Considering these points, risk management can be seen as a process with an emphasis on effective planning that uses the collection technique and data analysis. This article shows recommendations for project members so that they have the means to conduct the risk process better and prepare themselves facing uncertainty that surrounds the project.

In this context, uncertainty in projects has been the subject of some researchers' studies: [8] presents uncertainty as the lack of knowledge on the introduced innovations; [9] states that uncertainty is about our information state on the project goals, its job and its environment; [10] points out that uncertainty represents a threat, but we can not be sure what form it takes, otherwise we would identify it as a risk; Marinho et al. [11], [12] uncertainty in projects is defined as the lack of information and an inability to define the probability of an event to happen.

Uncertainty may arise from deficiencies in a range of knowledge areas, such as the contextual information on a project, our comprehension of underlying processes, explanations of past events, and the velocity of change (or time). We believe that uncertainty in projects results from limitations in seeing signs that may affect a project's success. Thus, it cannot be obtained through an occurrence probability, even subjectively. This difficulty may be generated by lack of experience, information, perceptive ability, or even due to the mindset of the people involved in the project. At this point, the organizational culture can have a strong influence.

In other words, it is believed that uncertainty in projects arises from individual experience (e.g., manager, team members') such as lack of knowledge, understanding and/or awareness of the project's important elements, its environment, and their interrelationship, so that one cannot obtain the probability that might impact the project's success. 
The purpose of this paper is to discuss some relevant aspects related to uncertainty management through semi-structured interviews with software project managers and management researchers. In particular, we aim to understand the practices that positively influence project management in face of uncertainties and to use the knowledge acquired during this study to provide recommendations that can be implemented by the industry to improve organizations' performance and their projects.

Besides the introductory section, this paper is structured as follows: Section 2 presents the literature review; Section 3 discusses the research method; Section 4 explores the interviews with specialists and the results from the semi-structured interviews; Section 5 presents the focus group data; Section 6 discusses the strategic orientations for uncertainty management; Section 7 presents a discussion of the results; and finally, Section 8 contains the conclusion.

\section{Literature Review}

This section discusses the concepts related to the work, namely early signs, and mindfulness.

\subsection{Early Signs}

In the mid-1970s, Igor Ansoff introduced the first version of his theory of weak signals [13]. Ansoff sought improvement to the strategic planning method, which does not work well when there are sudden changes or unforeseen discontinuities in business development. Ansoff states that strategic surprises can provide information before they happen, that is, there are signs or symptoms of surprises to come. While this information is initially inaccurate and the signs are vague, distorted, and difficult to interpret, these gradually become more distinct and easier to decipher.

Ansoff's starting point is the information a company receives from its environment. The information, knowledge accuracy and what is about to happen [14]. The terms used to refer to these signals are varied: early sign [15], [16], first warning sign [17], symptom, weak signal [13], [14], future signal [18], and warning sign [19]. A content comparison of these different terms shows that the authors refer to identical or very similar phenomena. The terminology used in this study is "early sign".

In a project's context, these early signs are of great importance, especially in innovative projects due to its various associated uncertainties. [15] address the issue in the project management context. The authors studied a number of early signs that have been identified in interviews by project managers. On the other hand, [20] evaluated the use of early warnings in research and development projects in Spain. [17] made an extensive research in the literature to develop a preliminary list of early signs.

Figure 1 presents the early signs groups based on the work of [15], [20], and [17] which were the early signs perceived by project managers, described below:

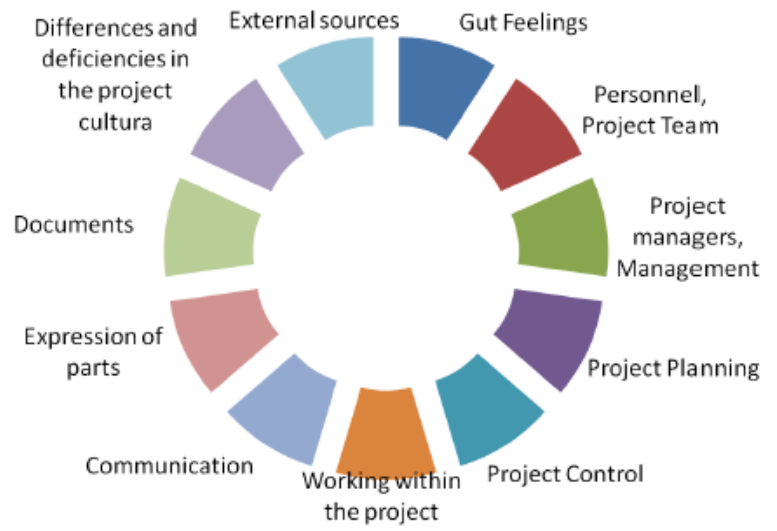

Fig. 1. Early signs groups. 
Gut feelings: Intuitive feelings; Personnel, project team: Nonverbal information, people's behaviour, lack of contact with the client, unrealistic planning, change of people, lack of professional skills; Project manager, management: Lack of leadership with the team and communication with clients, poor training, and lack of experience; Project Planning: Project connection with organizational strategy; Project Control: Progress control and monitoring; Working within the project: Early work, mobilization, repeated actions, and organization type; Communication: Communication between departments during the project; Expression of parts: Lack of CEO support, lack of decision, trust disappearance, and late recruitment; Documents: Quality, level, reports delivery time, changes in technical plans, incorrect revisions, and lack of clarity in responsibilities; Differences and deficiencies in the project culture: First impression, terminology in projects, lack of experience, and specific culture of a nation; External sources: External Sources and government regulations.

However, only the early signs group evaluation was not enough; we came across an article [21], [22], which stated that during the development of already known practices in project management, it is possible to detect early signs, but there was no instruction on how the project manager could improve the perception of early signs. In this case, we found in the literature, especially from the articles collected in the systematic review, how the project manager could be more aware of early signs and we came across the work of [23].

For early signs anticipated recognition, it is necessary to establish a mindfulness culture as well as check the early signs groups. The following subsection presents a discussion on this aspect.

\subsection{Mindfulness}

Weick and Sutcliffe use the term mindfulness to describe an alert state of mind for unexpected situations. The idea comes from an analysis of high reliability organizations (HROs) facing particularly difficult challenges in uncertainty management. These organizations conduct complex operations and operate in highly unpredictable environments where the potential for error can have very serious consequences.

Mindfulness is a comprehensive and holistic approach containing uncertainty in typically high uncertainty environments. HROs have cultivated mindfulness because in case of any failure, its proportions are catastrophic and simply unacceptable. [23] found that successful HROs tend to share five key attributes, as follows:

- Failure Concern: to find signs of emerging unexpected events, you have to look for them. The best way to do this is worrying about the failure possibility, being aware of its early signs, questioning whether there are different explanations for seemingly obvious results.

- Reluctance to simplify interpretations: as project managers struggle to understand what is happening within the project, there is a natural tendency to look for evidence to support preconceived ideas and reject what does not fit. However, all items of evidence must be considered on their own terms.

- Sensitivity to operations: early signs tend to be subtle and their insignificance is easy to ignore. Consequently, problems may erroneously be detected, often for some time before its negative aspects attention attraction. Even if the transition period is over, the problem effects are of low amplitude and difficult to realize without superb surveillance. Unless the team is sensitized and highly attuned to such anomalies, the clues are lost until the fault is no longer latent and materialize into a full problem.

- Commitment to resilience: A commitment to resilience means recognizing that any project aspect may be subject to uncertainties. There are no ways out of bounds. Of primary importance is that the team is ready and willing to face any uncertainty symptom as soon as it is detected. 
- Skills Consideration: Whenever a quick action is needed, trust becomes important. The project manager must be prepared to trust the team members to make important decisions. For this to work, there must be a shared understanding by all staff of the project objectives and the threat posed by uncertainty.

\section{Research Method}

Qualitative research is an inquiry method whose aim is to attain an in-depth understanding of human behaviour and the reasons that govern such behaviour [24]. It allows researchers to examine certain phenomena, develop insights, and report those insights. It involves discovering and understanding causes within a context. In qualitative research, the phenomena can be interpreted in a variety of ways, as it entails an interpretative evaluation [25]. The research method used in this work is based on the evidence-based software engineering principles which is based in conducting primary and secondary studies in different investigation stages [26].

Evidence-based software engineering aims to provide means by which the best evidence from research can be integrated with practical experience and human values in the decision-making process considering the development and software maintenance [26]. The essence of evidence-based paradigm is systematically collect and analyse all available data about a phenomenon for a more comprehensive and broader perspective than one can capture through a single study. This systematic approach, the method adopted, it is described in Figure 2.

First, an exploratory literature review [27] was performed with the aim of identifying the basic concepts and the main research sources in the area of domain and its results are summarized in [11]. Once the key research concepts were identified, scientific evidence to allow the analysis of the area of domain maturity was obtained.

Having identified, analysed, and understood the key research concepts, the following activity is to obtain scientific evidence to facilitate the analysis of domain maturity. According to [26], one of EBSE search procedures may be holding a systematic literature review. In this scenario, a research protocol was prepared and carried out in order to obtain and analyse the possible outcomes of preliminary studies conducted and published in a research area [12].

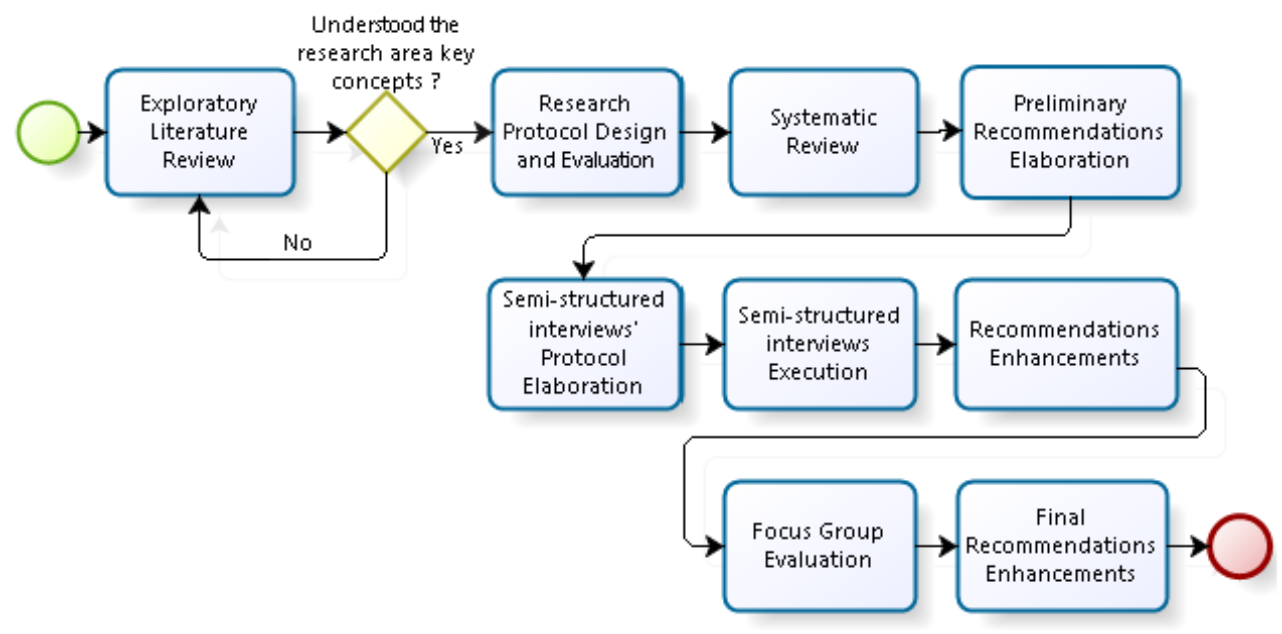

Fig. 2. Adopted method.

The first three steps enabled the identification of a set of recommendations addressing the project management in face of uncertainty. Among them, the importance of be aware of early signs to manage 
software projects.

When the recommendations are formulated, it is advisable to conduct researches with industry experts in order to obtain evidence items and possible improvements for it. At this point of the methodology, it was still necessary to expand search for other projects and other managers. Therefore, a series of semi-structured interviews were conducted to evaluate the viewpoint of other projects and managers, as well as the validity and importance of the recommendations.

A semi-structured interview focuses on a subject for which we made a script with the main questions that were complemented by other questions related to momentary interview circumstances. In addition to that, [28] claim that this approach could result in emergence of unexpected and clarifying information thus, improving the findings. Moreover, by using the interviews as data collection, they enable the researcher to get feedback on a particular research topic. The planning steps, the sampling and results are exhibited the next section. Therefore, the recommendations were revised in order to introduce enhancements.

Furthermore, a focus group with project management experts was conducted. The focus group is adopted to support researches in different areas, offering instruments to simultaneously collect qualitative data from a group of people [29]. In this work, the focus group was applied in the evidence-based software engineering context to obtain a proposed recommendations' evaluation. This step is also better explained later in the paper.

Finally, in order to guarantee the truthfulness of this work, and to be able to rely on its results, it was important to address from its planning, the necessary validity and reliability assessments. One of the most important ways to help confirm a qualitatively generated proposition is to ensure the validity of the methods used to generate it Seaman [28]. The validity of a study denotes the trustworthiness of the results, to what extent the results are true and not biased by the researchers subjective point of view [30].

The perspective adopted in the assessment followed Merriam and Tisdell [31]. The internal validity, the reliability, and the external validity were planed and analysed.

- Internal Validity: related to the issue of whether the results consistently represent reality [31].

- Reliability: this aspect is concerned with to what extent the data and the analysis are dependent on the specific researchers [30].

- External validity: This aspect of validity is concerned with to what extent it is possible to generalize the situation, and to what extent the findings are of interest to other people outside the investigated case [30].

The strategy used across this study was to (i) transcription by a third party and presentation to other researchers; (ii) use of protocol for conducting the systematic review, and interviews, the use of coding and Atlas.ti for syntheses and analysis, and the availability of the transcriptions for verification by other researches involved in the study; (iii) explicit research method and protocol and the publication of partial data.

\section{Interviews with Experts}

In this section, the summary of participants, sampling, data collection, and data analysis are presented. Initially, a semi-structured interview protocol (see in https://goo.gl/5RXCAL) with a set of questions was developed to guide the interviews. The project managers were emailed and informed about our research so that they could agree being interviewed or not. Face-to-face interviews were conducted with the participants using open-ended questions. All data during a specific data collection phase was collected and analysed in a subsequent data analysis phase. Key points from the data were collated and then assigned a code. The codes are characterized by evidence items here within quoted text. ATLAS.ti ${ }^{1}$ was used for quotes extraction and classification. 


\subsection{Participants}

In the research design, we planned that our target audience should involve innovative project managers, software managers, PMO managers, and researchers in project management. The researchers either were part-time project managers in innovation software projects or used to be project managers in such projects. Furthermore, researchers added their perspectives to this study based on different project management methodologies that they apply in their projects.

A total of 25 individuals with professional or academic experience in project management were interviewed. Among the interviewees, 4 were researchers from the project management area and 21 were project managers involved with information technology. Among the 21 project managers, 6 were working with innovative software projects, 8 were working with software projects, and 7 were responsible for the Project Management Office (PMO). Qualification of interviewees: 1 is postdoctoral, 3 hold a PhD, 13 have a master's degree and 8 have a Master's in Business Administration (MBA). The organizations' profiles from interview's sample were composed by 10 profit-making, 8 governmental organizations, 7 from non-profit organizations. The size of the organizations: 15 were large, 6 were medium, 1 was small, and 3 were micro enterprises. The Brazilian market in which the organizations operate: 14 national; 6 regional; 4 global and 1 multinational.

Table 1 presents a summary of the participants' data, which are going to be labelled by a P1 to P25 code. In Table 1, PM is the abbreviation for project management and RPM is research in project management. The professional experience level of the interviewees varies from 6 to 35 years and the time of involvement with project management ranges from 2 to 20 years.

Table 1. The Arrangement of Channels

\begin{tabular}{ccccc}
\hline \hline Code & Role & PM Experience & Education & Company Type \\
\hline P1 & PM & 19 & MSc Computing Science & Governmental \\
P2 & PM & 18 & PhD Computing Science & Private \\
P3 & PM & 7 & MSc Computing Science & Private \\
P4 & PM & 15 & Mba in Project Management & Private \\
P5 & PM & 13 & MSc Computing Science & Governmental \\
P6 & PM & 11 & MSc Computing Science & Nonprofit \\
P7 & RPM & 30 & PhD Computing Science & Private \\
P8 & PM & 16 & Mba in Project Management & Governmental \\
P9 & PM & 8 & Mba Software Engineering & Private \\
P10 & RPM & 2 & MSc Computing Science & Nonprofit \\
P11 & PM & 9 & MSc Computing Science & Private \\
P12 & PM & 2 & Mba Software Engineering & Nonprofit \\
P13 & PM & 10 & MSc Computing Science & Private \\
P14 & PM & 6 & Mba in Project Management & Nonprofit \\
P15 & RPM & 5 & PhD Computing Science & Private \\
P16 & PM & 9 & Mba in Project Management & Nonprofit \\
P17 & PM & 5 & PhD Computing Science & Private \\
P18 & PM & 10 & Mba in Project Management & Governmental \\
P19 & PM & 20 & MSc Computing Science & Private \\
P20 & PM & 12 & MSc Computing Science & Nonprofit \\
P21 & RPM & 8 & MSc Computing Science & Governmental \\
P22 & PM & 5 & MSc Computing Science & Governmental \\
P23 & PM & 5 & MSc Computing Science & Governmental \\
P24 & PM & 12 & MSc Computing Science & Governmental \\
P25 & PM & 10 & Mba Software Engineering & \\
\hline \hline & & & & \\
\end{tabular}

\subsection{Sampling}

One of the inevitable and sometimes uncomfortable methodological decisions in the qualitative research 
and interview work is the decision about who, how many people and how often interview. A common strategy to define the theoretical sample is the saturation or redundancy principle defined by [32]. According to these authors, the researcher must judge the closing of the sample according to the theoretical saturation of a subject. Saturation means that once similar cases start to be recurrent, the investigator acquires some empirical confidence that there is no additional data that may contribute to the development of research [33], [34].

\subsection{Data Collection}

Initially, a protocol (see in https://goo.gl/5RXCAL) with a set of questions was developed to guide the interviews. The project managers were emailed and informed about our research so they would agree or not on being interviewed. The interviews were scheduled at time and location interviewer and participant mutually agreed. Face-to-face and one-to-one interviews were conducted with the participants using open-ended questions. The participants were introduced to the early signs and mindfulness conceits. They were asked to comment on the mindfulness constructs and to describe how they worked with their team. They were voice-recorded for our convenience, with the participants' permission.

\subsection{Data Analysis}

All data during a specific gathered phase was collected and examined in a subsequent data analysis phase. The audio recording was an important practice to allow the interviewer to concentrate on the conversation. After that, each interview was transcribed and reviewed verbatim to explore the data meaning by searching for similarities and differences. Key points from the data were collated and then assigned to a code or a summary phrase.

The codes are characterized by evidence items presented in quotes. In order to carry out qualitative data analysis we have adopted the software ATLAS.ti ${ }^{1}$, helping us to perform quotes extraction, their categorization and classifications of the evidence items gathered. The codes arising from data were constantly compared to codes previously gathered. From the constant comparisons of the codes [35], we grouped codes into "categories" that represent factors affecting project uncertainty.

Despite of we recognize that the best way to identify and analyse behaviour in teamwork is the triangulation among different data collection source, we understand each evidenced concept as a category where fit several concepts related to the same pattern behaviour, which led us to synthesize our findings using constant comparative method of qualitative analysis onto data [36] and disciplined the imagination of [37].

\subsection{Interview Results}

In this section, the findings collected during semi-structured interviews are going to be presented. The interviews' findings are going to be shown according to protocol orientation. For each extracted quote, the following format was adopted: [QU participant number: sequence of the extracted quota].

Further, as an evaluation form of the concepts presented and treated during interviews; a network was elaborated, as shown in Figure 3. According to [38], a network is an assessment tool of a set of defined terms in a study. Figure 3 was developed through evidence extracted from the interviews' transcripts, it presents the concepts that were expressed by participants. It is worth highlighting that the evidence items are represented by a project manager's sentence portion to confirm the concepts; the answer types: yes or no are not considered in network.

Furthermore, Figure 3 depicts the Network Analysis carried out about the "early signal" mentioned explicitly or implicitly by interviewees. The first number presented in each node denotes the frequency of

${ }^{1}$ http://atlasti.com/ 
emerging evidence items (quotes) pointing out to each concept, whereas the second number describe the density of the network for each node, i.e., how many connections each concept has with other ones.

The network's central concept was early signs with 23 evidence items. For the mindfulness concept, 16 evidence items were found. The attributes to detect the signs presented a total of 103 evidence items divided as follows: Failure Concerns with 23; Reluctance to simplify interpretations with 20; Operations Sensitivity 20; Commitment to resilience with 29; and Skills Consideration with 14.

All the evidence was found from the interviews. The project management practices found 133 evidence items divided into: Risk analysis with 18; Earned value management with 17; Project evaluations with 19; Performance measurement with 21; Stakeholders' analysis with 22; Maturity assessments with 16; and Consultation from past projects with 20.

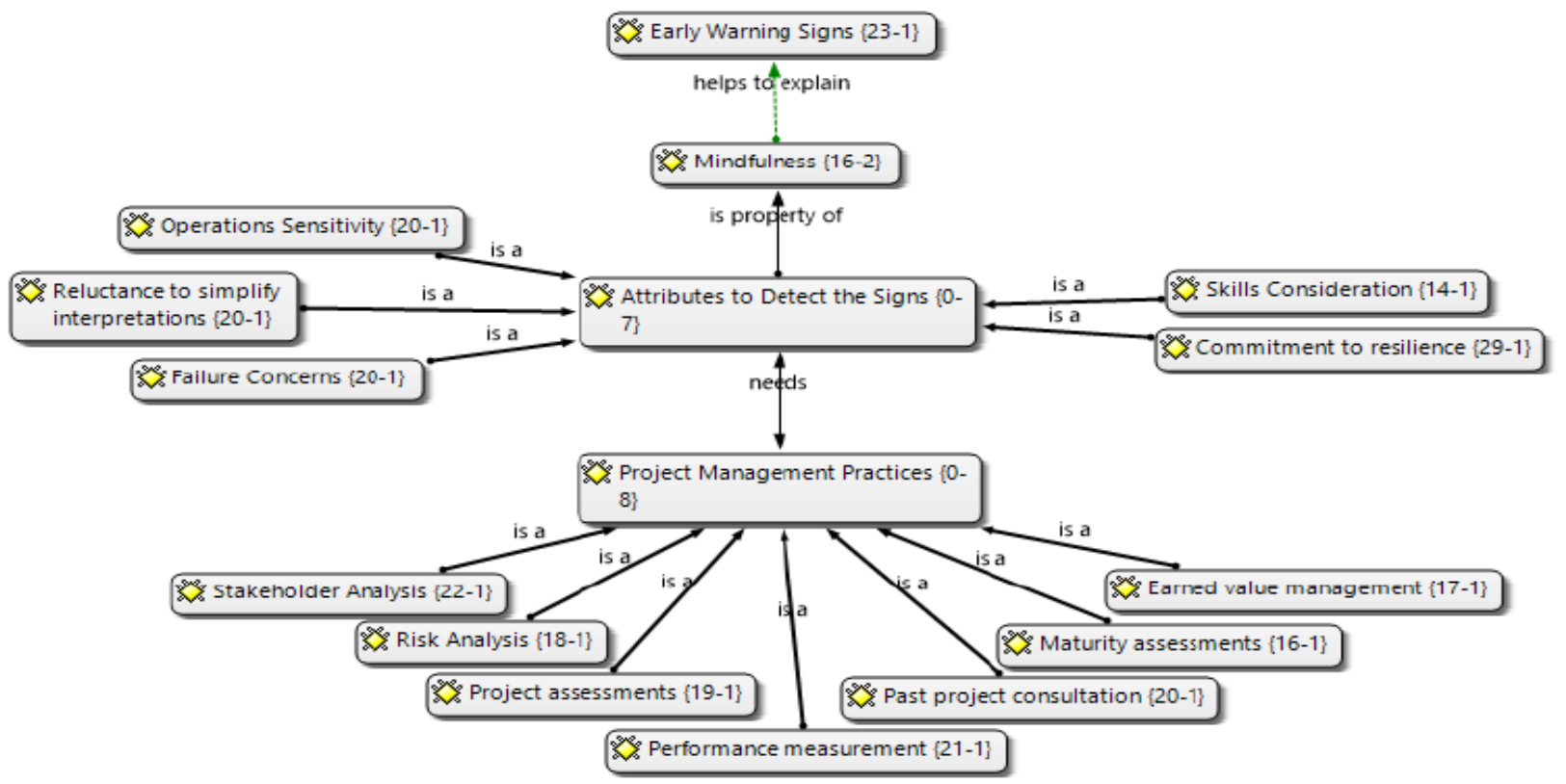

Fig 3. Early signs network.

However, it is necessary to point out that, all respondents indicated strong relationship between management practices and the attributes to detect the early signs, that is, without the attributes the use of management practices do not work properly to manage uncertainties in software projects. In addition, the attributes are considered a mindfulness property which helps explain the early warning signs. Furthermore, the participants interview's claim that the mindfulness attributes should be considered in innovative software projects and it makes sense to bringing the HROs concepts in order to better understand the uncertainties in software team context and to improve performances. The following subsections demonstrate fragments from interviews that helped to build the recommendations that guide managers on dealing with uncertainties.

\subsubsection{Early warning signs detection}

Based on the five attributes [23], the participants were asked whether they agreed with the actions to assess the projects' mindfulness level to early signs. In the first attribute Failure Concerns, the manager P14 commented on the fact of observing both the success of a project and the failure:

"You need to look at both sides, because if I do not manage the failure; I do not look at the failing possibility, so what? If I have failure signs, I do not have contingencies; and if I do not have contingencies, I'll fail." [QU14:11]. 
However, participant P12 warns that within his project the term failure is avoided:

"We do not use the failure, we set our goal, our goal is that... and we try to follow the goal." [QU12:5].

Still, manager P18 present a more open way, presenting both opportunities to the team:

"I have a custom when working with a team; I teamwork in a very open and proactive way. I love receiving feedback, suggestions, criticisms; conversely, I am quite clear and objective in our success chances and our failure possibilities." [QU18:16].

Nevertheless, P8 says that failure is a possibility that no one wants to face, and thus, one needs to face it:

"We align projects a lot and always think of the failure possibilities because we do not want to go towards that direction."[QU8:6]

Manager P17 adds that it is necessary for the team to have a coherent thought. It is more probable that, if we observe failure in a coherent way, getting help from the staff without being too negative can work well:

"If it's done in a systematic way so as not to create a totally pessimistic team, I think it can be touched; yes, such as follows: let's have a coherent thought, that everyone may have a more probable opinion." [QU17:9]

Only 2 out of 25 participants did not agree with this stage. It is believed that, according to what was exposed by other managers, if the failure concern attribute was done collaboratively, presenting both sides of the coin it would be better managed. It is important that it is shown by the manager that the team is part of the project's environmental context and while making use of this attribute, the teams will not be stressed with the comments focused on the failure.

Regarding the Reluctance to simplify interpretations, the 25 of them believe that this has to be done by all project managers. Participant P1 mention about the importance of observing several evidence items:

"All evidence must be observed. I tried to do this even without knowing; seeing these early signs." [QU1:19].

P18 show that in addition to observing, one must interpret the evidence the best way, trying to encompass all the possibilities:

"I should be aware of all the possibilities that this happens, we have to is working on this activity, trying to surround all the possibilities." [QU18:17]

In addition, P20 say:

"You have to work with reflection and consider everything and everyone." [QU20:7]

In the case of Operations Sensitivity, all also agreed with this stage. The necessity of this topic was unanimous. Many managers try to maintain a dialogue with the team as P12 say,

"When we notice that the business is not going well or a person is very unsatisfied, I try to talk to the person, investigate what's going on." [QU12:7].

P15 adds the fact that one's close to the team to understand everybody's perception:

"In the projects I'm working closest, I'd prefer to talk to people and understand each one's perception." [QU15:7].

In Commitment to resilience, out of the 25 interviewed, four did not agree with this attribute, such as manager P15:

"I think the team has to report the symptoms; if the team is analysing the symptoms they perhaps may have a distorted view of the facts." [QU15:9].

According the interview's excerpt and the researcher's feeling, manager P15, like the others who did not agree, are more traditional and centralizing managers. The majority that agrees with the attribute talked things such as follows:

"See that I was beginning to equip myself with information, but in the beginning it was not so, I had to create that culture in the team." [QU1:26];

"You create a culture to enable them to identify and longer cope with these uncertainties." [QU10:10].

In addition, $\mathrm{P} 2$ told that the team needs to be prepared and this requires giving the staff autonomy: 
"Innovation projects have uncertainties and it is necessary to know how to deal with them. When you train a person well, they are ready to face the uncertainties." [QU2:10]

P10 complement it saying that it is necessary to have the team prepared for frustration and that they need to have the maturity to deal with uncertainties:

"It is interesting for the team to have a high maturity degree and cope with uncertainty better." [QU10:13]

The attribute Skills Consideration, only 1 of 25 did not agree with this attribute. The other managers agree that the team members' experience should be taken into consideration.

"When I go through an experience, I absorb it and get smarter for the next time. The same thing has to happen with the team; they went through other experiences in other projects, so if they observe a particular problem in the current project, that may happen; I think they have to warn me. This is based on their experience." [QU6:16]

Alternatively, P21 presents that the divergence within the team is healthy and should be scored to reach a consensus:

"If there is anyone who is thinking that something else should be done, either that person did not understand what to do or the team did not see what that person is seeing, then the whole dispute has to be scored." [QU21:10]

Various software project management activities can be used to detect the early signs, but for doing so; managers and the team need to put into practice the mindfulness. As shown by several participants and some of the following evidence:

"We treat the importance of being always aware of this project, but we also explain to the team the following: the success this project does not depend only on you. It will depend on other people and that is why it is important to pay attention on the other people's actions." [QU22:12]

"I think mindfulness is fundamental to look for these signs, because that's how, in fact, we anticipate ourselves before the problem materialize. When you see, you can feel a lot. With sensitivity you realize before; even by the team's voice tone." [QU23:13]

Hereinafter practices that have been mapped in [21] and are familiar to project management which can be applied in the context of identifying early signs will be presented. The participants were asked whether they agreed that one can detect early signs through the presented practices.

The interviews also presented the use of some practices that could help in the detection of early warning signs, such as risk analysis, earned value management, project evaluations, measurement performance, management stakeholders, maturity assessments and past project consultation.

In addition to these activities presented, if the project manager is always using mindfulness, he will be able to capture the early signs in any activity, as spoken by P23:

"Anytime we talk, you interact if you use mindfulness, then you catch something." [QU23:17]

A concern that manager P23 had is related to a project manager's reflection moments. The manager has always many activities and begins not to notice the signs, as shown below:

"The step in which you have been looking at a particular aspect, will shed light on that aspect; you will reflect on something and you can find signs there, it's difficult to have those moments and to enable the project manager mindfulness. Because you're usually doing five things simultaneously." [QU23:19]

We agree with the P23, who clearly said that if the project manager takes mindfulness it will be able to detect the early signs in any project activity.

\section{Focus Group}

From the interviews and the project managers' feedback, the orientation was structured, keeping the initial stages. Thus, the focus group was conducted to evaluate the proposed recommendations. The focus 
group main aim is to identify participants' feelings about a certain matter, product, or activity. Its specific objectives vary according to the research approach. In an exploratory research, its purpose is to generate new ideas or hypothesis and stimulate the researcher's thinking, while in phenomenological or orientation research, it is to learn how the participants interpret the reality, their knowledge and experiences [29].

Six innovative software project managers of companies in Brazil with an average of 20 years of professional experience took part in the focus group. Forms were designed for each recommendation to help evaluate each stage of it, as well as slides for driving every step. During the execution, the meeting was three hours and a half long. The Focus Group meeting took place in a traditional classroom, with a data projector for presenting slides and fixed seat rows, which is not an ideal setting for the planned dynamics. However, it was possible to accommodate the participants and make the dynamics work.

The dynamic worked as follows: first, the participants were presented. The moderator introduced the focus group rules generally and as well as every recommendations step. The forms were distributed so that at least three participants would evaluate the activities. At the end of each assessment, the participants had to write the activity on a post-it individually and then stick it on a frame (made of cardboard) containing the recommendations' steps (Evaluating whether mindfulness attributes are being used and Checking the early signs group) indicating in which moment the recommendation should be held and suggesting (in forms) improvements for each of them.

Regarding the participants' evaluations, all recommendations evaluations were positive and they said that the recommendations quantity was necessary for uncertainty management. A structure which was formalized in the focus group will be presented in the next section.

\section{Recommendations to Manage the Uncertainties}

Once it is impossible to predict the problem nature in advance, project managers can employ strategies that impregnate their projects with greater resistance. Throughout a project, a series of practices can be established in order to keep uncertainties managed. The orientation was created based on the literature and practice evidence. For early signs anticipated recognition it is necessary to establish a mindfulness culture, as well as check the early signs group. Figure 4 shows the activities to help identify early signs.

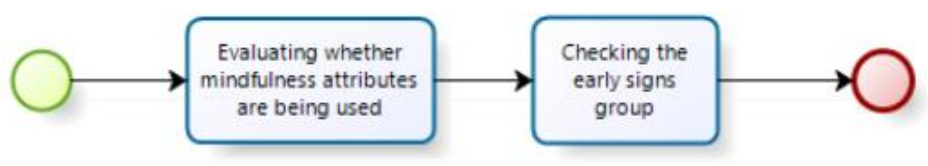

Fig. 4. Detecting early signs.

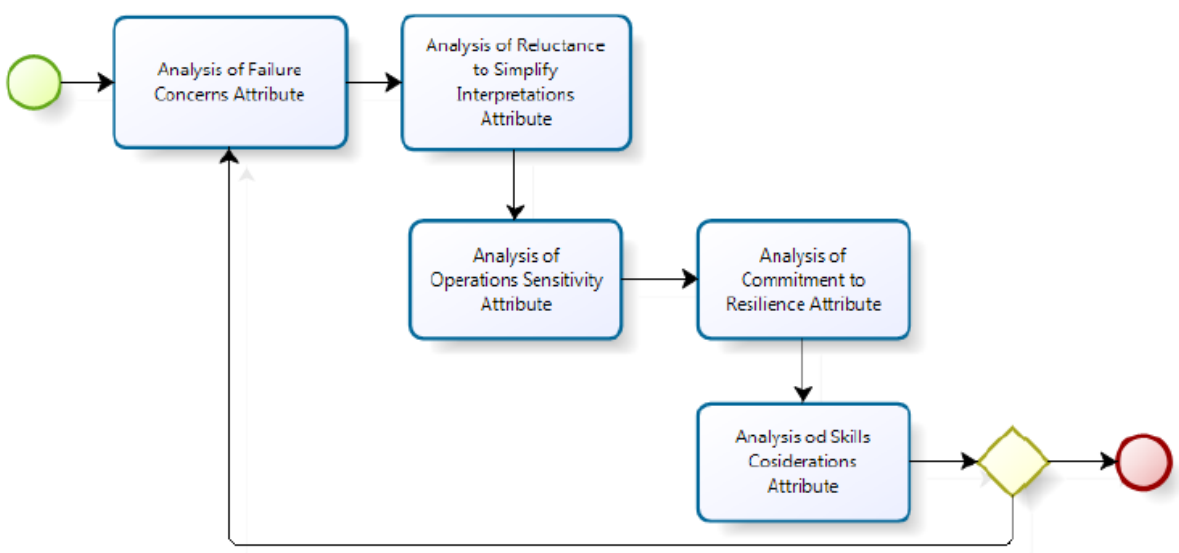

Fig. 5. Evaluating whether mindfulness attributes are being used. 


\subsection{Evaluating Whether Mindfulness Attributes are Being Used}

For being able to detect early warning signs in their projects, the manager needs to use the mindfulness concept. In this study, through the evidence found, it is presented a way the team members may reflect on mindfulness in the project context. In addition, some guidelines are suggested. Fig. 5 depicts the activities.

The mindfulness concept can be established through five attributes based on evidence items that are presented below:

Failure Concerns: In order to find the signs it is necessary to watch out for them questioning whether there are different explanations with seemingly obvious results. The best way to accomplish that is to make the project team aware of the failure possibility.

To verify if the failure concerns are well applied in the project. The manager and the team should reflect on the following questions:

1) Does the team always look at the flaws and try to understand them?

2) When something unexpected happens, does the team always try to find out why the expectations were not met?

3) Does the team consider the early signs as information and try to learn from them?

4) Does the team consider the early signs as points that reveal potential dangers and not successes that demonstrate the ability to

avoid disaster?

5) If a team member makes a mistake, is not this error used against them?

6) Do the team members report significant errors even if others do not realize that mistakes are made?

7) Do managers actively look for early signs?

8) Do team members feel free to talk to superiors about problems?

9) Are team members rewarded if they detect early signs or potential problematic issues?

The more positive responses, indicates that the project team is healthily concerned with project failure. The manager must use these questions to start thinking about ways to improve the project attribute application. Some actions are recommended:

- The manager should foster the team reflective ability;

- The manager should foster an appreciative approach to deal with mistakes;

- The manager should sensitize the team members about the errors possibility so that they feel responsible and attentive to the signs;

- The manager must create a learning culture for everyone to share mistakes and experiences;

- The manager should fostering self-organizations, team cohesion, team spirit while introduce a critical approach to handle with failures;

- The team should review the projects goals and pay attention to the mistakes that should not occur.

Reluctance to simplify interpretations: The project manager struggles to understand what is happening within the project and there is a natural tendency to look for evidence to support preconceived ideas and reject what does not fit. However, all evidence must be considered;

To verify if the reluctance to simplify interpretations attribute is well applied in the project. The manager and the team should reflect on the following questions:

1) Do team members strive to challenge the status quo?

2) Do team members feel free to bring problems and difficult issues?

3) Do team members usually deepen their analysis to better understand the uncertainties in projects?

4) Are team members encouraged to express different world views?

5) Do team members listen carefully, and rarely someone's vision is not heard? 
6) Are not team members punished when they report information that could disrupt operations?

7) When something unexpected happens, do team members spend more time analysing than defending their vision?

8) Are sceptics highly valued?

9) Do team members trust each other?

10) Do team members show respect for each other?

The more positive responses, the more the project uses the reluctance to simplify interpretations attribute. The manager must use these questions to start thinking about ways to improve the project results. Some actions are recommended:

- The team must raise doubts to gather information: Try to look beyond the limits of their expectations;

- The manager should encourage mutual respect to differences so that everyone can voice their opinions;

- The manager should make the team think under other perspectives.

Operations Sensitivity: The early signs tend to be subtle. Their trifles sometimes make them easy to ignore. Therefore, many problems may remain undetected. The entire team must be ready, alert to detect, monitor, analyse, and determine if there really is an uncertainty associated with the identified sign;

To verify if the Operations Sensitivity attribute is well applied in the project. The manager and the team should reflect on the following questions:

1) In day-to-day, is the manager always paying attention to what's going on?

2) When problems happen, is someone with authority to act always accessible to team members?

3) Do team members have the power to solve unexpected problems that might arise?

4) During a normal day, do team members interact frequently enough to build a clear picture of the project's current situation?

5) Are team Members always looking for feedback on things that are not going well?

6) Are team members familiar with the operations beyond their own functions?

7) Do managers constantly monitor workloads and reduce them when they become excessive?

The greater the number of negative responses is, the less is the sensitivity to the operations. The manager must use these questions to start thinking about ways to improve the sensitivity for operations. Some actions are recommended:

- The manager should constantly stick to the information passed by the team, whether verbal or not;

- Team members should speak. Just because one member noticed something, one should not assume that the others noticed too, it is important to communicate;

- Team members must develop the ability to be sceptical: When you are sceptical, it is likely to better assess the activities carried out and the comments raised can support or disprove a certain activity;

- The manager should provide feedback and encourage people to listen;

- The manager should spend time with team members following the daily work.

Commitment to resilience: It is recognizing that any project aspect may be subject to uncertainty. There are no off limits aspects. All that matters is that the team is ready and willing to face any uncertainty symptoms as soon as they are detected;

Resilience is the ability to withstand and cope with unexpected situations, it is a key feature for project teams involving many uncertainties. Resilient teams tend to find solutions to everyday challenges.

To verify if the commitment to resilience: attribute is well applied in the project. The manager and the team should reflect on the following questions:

1) Do most team members have skills to act on the unexpected problems that might arise?

2) Do team members learn from their mistakes? 
3) Are there resources to training and continuous recycling of team members?

4) Do team members have more than enough training and experience for playing their role in the project?

5) Are project leaders actively concerned with the team members' skills and knowledge development?

6) Are the team members known for their ability to use their knowledge in an innovative way?

7) Is there a concern with team members' skills building?

8) Do team members have an informal contact network they may sometimes use to solve problems?

9) Do team members trust each other?

The greater the number of positive answers, the better for the project, as it shows a resilient team. If points such as these are not applied in the project context, the manager and team need to reflect on how to improve the detected points. Some actions are recommended for the project to take into consideration the attribute commitment to resilience; they are as follows:

- Accept that unpleasant situations and uncertainty are part of the project;

- Believe and nurture skills and team skills to deal with difficult situations and develop emotional intelligence;

- Always nurture team confidence, especially regarding the belief that one is able to achieve goals;

- Learn to keep calm in all situations;

- Always find the positive and even fun side of stressful situations;

- Value the maturity of the team.

Skills Consideration: When a problem arises within the project, experts in a given subject can be the best strategy to solve them, although other members should not simply push the problem to the expert, instead of that the team must try to learn with the expert and the problem resolution. To verify if the attribute is applied in the project. The manager and the team should reflect on the following questions:

1) Is the team committed to do their job?

2) Does the team respect one another's activity nature?

3) If something unusual happens, does the team know who has the knowledge to respond to it?

4) Do the team members value expertise and experience on the hierarchical level?

5) In the project, do the most qualified people to make decisions make them?

6) Do team members usually become problem owners until it is resolved?

7) In general, is it easy to obtain expert assistance if something comes up that the team does not know how to handle?

The larger the number of positive answers, the better for the project, because it shows that there is concern in applying the attribute. If points such as these are not applied in the project context, the manager and team need to reflect on how to improve the detected points.

Some actions are recommended for the project to take the attribute into consideration the Skills Consideration; they are as follows:

- Beware of the centralization fallacy: The manager needs specialists to think realistically. It is necessary to let each one act autonomously within the project;

- Stimulate the imagination as a tool to manage uncertainty: Facing uncertainties, it is necessary to use the imagination. The use of scenarios may be an ally in the search for possible solutions;

- Create flexible decision-making structures: Do not assume that the expertise is at the top of the hierarchy. When there are uncertainties or problems occur, try to divert to who can really help.

The manager must put into practice the five attributes in the day-to-day of the project in a way to stimulate the team integration based on the present moment experience, fully aware with an open, 
non-judgmental attitude in every activity performed.

\subsection{Checking the Early Signs Group}

During the project cycle the manager can make use of the early signs group presented in Fig. 1 in order to guide them. Fig. 1 presents the main early signs groups identified and some scholars' description [15], [17], [20]. In addition to the signs shown in Figure 1 other signs can be seen during a project's course. The manager can use the five attributes to watch them.

\section{General Discussion}

According to the evidence gathered during development of this study is still a nascent research topic, because as advocated by [39], the large number of exploratory qualitative studies denotes that the studies on this area are still maturing. As a consequence, this condition implies a demand for exploratory qualitative studies, originally open-ended data that have to be construed for meaning: what matches with the evidence profile found in this study. Notwithstanding, as a natural development for this trend topic, in further studies we plan apply a mixed method approach for future work.

However, the mindfulness establishment by grouping the five attributes for early signs detection, in the context of innovative software projects, were evaluated resulting in a series of recommendations. Furthermore, we designed a focus group in order to evaluate the recommendations and to suggest possible improvements. The focus group was useful to better structure the recommendations and to verify the need for each recommendation. The focus group participants reached a consensus that the recommendations are necessary for the industry to improve organizational performance through their projects.

The idea of recommendations is to help innovative software development organizations achieve better results, while remaining competitive, enhancing their team skills and to do not let their projects be predestined to failure. In order to go beyond the "off the shelf solutions" the recommendations help guide and train reflective practitioners to rethink the status quo and overcome their problems. We encourage management thinking that inspires different ways of thinking, reflecting and learning to deal with the uncertainties.

Regarding the validity of the work there are several options to develop an assessment concerning to the strength of evidences in Atkins et al. [40]. Across this study, the internal validity, the reliability and external validity were analysed as described in section 2 . The discussion on this analysis is presented in Table 2 .

Table 2. Threats to Validity

\begin{tabular}{ll}
\hline \hline \multicolumn{1}{c}{ Validity } & \multicolumn{1}{c}{ Discussion } \\
\hline \multirow{3}{*}{$\begin{array}{l}\text { Internal } \\
\text { Validity l }\end{array}$} & $\begin{array}{l}\text { The interview was recorded and the transcriptions were faithfully by a third party, some quotes were } \\
\text { presented across section 4.5. Sampling participants with maximum variation, followings revised and approved } \\
\text { protocol, using literature data from SLR. In order to increase the internal validity, Atlas.ti was used to analysis } \\
\text { and synthesis. }\end{array}$ \\
As previously said, the interview used a third party to transcript the taped interviews and used codes analysed \\
in Atlas.ti. In order to cross the affirmations with the literature review, the SLR data were also synthesized and \\
analysed with the help of the Atlas.ti. \\
The validity was addressed by transparency of the tested instruments and protocols. Independent researchers \\
were always involved on each step to verifications. Further, each step along the study was also published. As a \\
Validity & $\begin{array}{l}\text { result of the combined synthesis, a deeper investigation with six innovation software project management } \\
\text { were performed, comparing and cross-analysing the data that pointed out the set of recommendations } \\
\text { presented in this paper. }\end{array}$ \\
\hline \hline
\end{tabular}

It is important to notice that some quotes seem to fit with the patterns expected by our inquiry. It leads Us to think about strength of evidence and study limitations.

In keeping with Kitchenham et al. [41], some of them propose that the strength of evidence can be 
established on a hierarchy where concreteness from randomized experiments and systematic reviews at the apex of this hierarchy and evidence from expert opinion at the base of this hierarchy. Notwithstanding, setting up this kind of experiments sometimes demands a high time consumer or are not feasible in specific contexts. In these cases, the application of observational studies can be a good alternative to produce better evidence.

In relation to the interviews with the sample of project management professionals, 25 Brazilian professionals participated in these interviews. This number of professionals influences the generalization of the final results, as well as the focus group participants. Here there is a limitation of Brazilian professionals only. Moreover, another limitation is that the recommendations have not been applied yet in a software development project, but it is an opportunity to contribute to the continuity of the developed studies as well as verify the effectiveness of their findings.

\section{Conclusion}

Project management has a number of foundations to support the project managers. This study has aimed to provide additional recommendations to these tools and techniques as well as raised some reflections to use them as opportunities to perceive early signs and act with the project uncertainties. This study was built on evidence-based software engineering and it provides subsidy for the need to address uncertainty in software projects in order to reduce the negative impacts caused by them. Further, it contributes by defining recommendations to uncertainty management.

In order to deal with the lack of information and an inability to define the probability of an event to happen, the project manager must become aware of the early signs that may affect a project success. Uncertainty is a dimension that software project managers should observe for improving projects' implementation.

While investigating the literature and seeking a way to manage uncertainties, the early signs and mindfulness were found to be profusely related. During the interviews, it could be noticed that the concepts that were detected in the first two stages of the methodology was solidified by the project managers' assessments, as there was an acceptance of the parties addressed throughout the interview; it was also stated that the concept of managing uncertainty makes sense for software project managers. During the interviews, it was found that this concept is fully connected to (1) observing the project continuously so that through the members' perception threats may be identified; (2) adopting strategies to be able to reveal risks; (3) preparing the team with mindfulness to deal with the unexpected; (4) obtaining greater knowledge and preparing projects to better face the unknown and proactively manage it so that the project is prevented from harmful events.

Among the difficulties faced along this study, the overwhelming amount of data collected during the interviews is outstanding. For every hour of audio's interviews, at least another two hours were spent for coding on Atlas.ti and for analysing the emerging evidence. Moreover, the limitations are the same as in any qualitative research, as culture is always evolving; the findings are context and time dependent. In our study, the context was the Brazilian industry, since both the focus group and the interviewees sample comprised only Brazilian professionals.

Based on the interview findings, this study presented a series of recommendations that may be established in order to manage uncertainties throughout a project. The mindfulness concept and its use can be used along with those recommendations. Such recommendations give instructions that can be used to detect early signs during the development of already well-known practices in project management. Moreover, it can help the project manager to improve his/her early signs' perception. 


\section{Acknowledgment}

Authors would like to thank Postgraduate Programme in Administration and Rural Development PADR for supporting the development of this work as well as the reviewers.

\section{References}

[1] Marinho, M. L. M., Sampaio, S. C. B., Lima, T. L. A., \& Moura, H. P. (2015a). Uncertainty management in software projects. Journal of Software, 10(3), 288-303.

[2] Marinho, M. L. M., Lima, T. L. A., Sampaio, S. C. B., \& Moura, H. P. (2015b). Uncertainty management in software projects an action research, Proceedings of the XVIII Ibero-American Conference on Software Engineering.

[3] Zaina, L. A., \& A' lvaro, A. (2015). A design methodology for user-centered innovation in the software development area. Journal of Systems and Software, 155-177.

[4] Godoi, R., Marinho, M., \& Moura, H. (2015). Narrowing impact factors for innovative software project management. Elsevier.

[5] Bannerman, P. L. (2008). Risk and risk management in software projects: A reassessment. Journal of Systems and Software, 81(12), 2118-2133.

[6] O'Connor, G. C., \& Rice, M. P. (2013). A comprehensive model of uncertainty associated with radical innovation. Journal of Product Innovation Management.

[7] Perminova, O., Gustafsson, M., \& Wikstr"om, K. (2008) Defining uncertainty in projects-a new perspective. International Journal of Project Management, 26(1), 73-79.

[8] Loch, C. H., DeMeyer, A. \& Pich, M. T. (2011). Managing the unknown: A new approach to managing high uncertainty and risk in projects. John Wiley \& Sons.

[9] Shenhar, A. \& Dvir, D. (2007). Reinventing project management: The diamond approach to successful growth and innovation. Harvard Business Press.

[10] Cleden, D. (2009). Managing project uncertainty. Gower Publishing, Ltd.

[11] Marinho, M. L. M., Sampaio, S. C. B., \& Moura, H. P. (2013). An approach related to uncertainty in software projects. Proceedings of the 2013 IEEE International Conference on Systems, Man, and Cybernetics (SMC).

[12] Marinho, M. L. M., Sampaio, S. C. B. Lima, T. L. A., \& Moura, H. P. (2014). A systematic review of uncertainties in software projects. International Journal of Engineering \& Applications.

[13] Ansoff, H. I. (1975). Managing strategic surprise by response to weak signals. California Management Review.

[14] Ansoff, H. I. (1984). Implanting strategic management. Prentice/Hall International Inc. USA.

[15] Nikander, I. O., \& Eloranta, E. (2001). Project management by early warnings. International Journal of Project Management.

[16] Nikander, I. O., et al. (2002). Early warnings: a phenomenon in project management. Helsinki University of Technology.

[17] Kappelman, L. A., McKeeman, R., \& Zhang, L. (2006) Early warning signs of it project failure: The dominant dozen. Information Systems Management.

[18] Hiltunen, E. (2008). The future sign and its three dimensions. Futures.

[19] OGC. (2009) Managing successful projects with PRINCE2. The Stationery Office.

[20] Sanchez, A. M. \& Leybrne, E. (2006). Early warning signals for r\&d projects: An empirical study. International Journal of Project Management.

[21] Haji-Kazemi, S., Andersen, B., \& Krane, H. P. (2013). A review on possible approaches for detecting early warning signs in projects. Project Management Journal. 
[22] Haji-Kazemi, S., Andersen, B., \& Klakegg, O. J.(2015) Barriers against effective responses to early warning signs in projects. International Journal of Project Management.

[23] Weick, K. E., \& Sutcliffe, K. M. (2011). Managing the unexpected: Resilient performance in an age of uncertainty. John Wiley \& Sons.

[24] Denzin, N. K., \& Lincoln, Y. S. (2005). The sage handbook of qualitative research.

[25] Creswell, J. W. (2013). Qualitative inquiry and research design: Choosing among five approaches. Sage.

[26] Kitchenham, B. A., Dyba, T., \& Jorgensen, M. Evidence-based software engineering. Proceedings. 26th International Conference on Software Engineering.

[27] Schuetzenmeister, F. (2010). University research management: An exploratory literature review. Institute of European Studies.

[28] Seaman, C. B. (1999). Qualitative methods in empirical studies of software engineering. Software Engineering, IEEE Transactions on.

[29] Shull, F., Singer, J., \& Sjøberg, D. I. (2008). Guide to advanced empirical software engineering.

[30] Runeson, P., \& H"ost, M. (2009). Guidelines for conducting and reporting case study research in software engineering. Empirical software engineering.

[31] Merriam, S. B., \& Tisdell, E. J. (1015). Qualitative research: A guide to design and implementation. John Wiley \& Sons.

[32] Glaser, B. G., \& Strauss, A. L. (2009). The discovery of grounded theory: Strategies for qualitative research. Transaction Publishers.

[33] Dorairaj, S., Noble, J., \& Malik, P. (2011). Bridging cultural differences: A grounded theory perspective. Proceedings of the 4th India Software Engineering Conference.

[34] Adolph, S., Kruchten, P., \& Hall, W. (2012). Reconciling perspectives: A grounded theory of how people manage the process of software development. Journal of Systems and Software.

[35] Corbin, J., \& Strauss, A. (2008). Basics of qualitative research: Techniques and procedures for developing grounded theory. Thousand Oaks: California.

[36] Glaser, B. G. (1965). The constant comparative method of qualitative analysis.

[37] Weick, K. E. (1989). Theory construction as disciplined imagination. Academy of Management Review.

[38] Cronbach, L. J., \& Meehl, P. E. (1955). Construct validity in psychological tests. Psychological Bulletin.

[39] Edmondson, A. C., \& McManus, S. E. (2007). Methodological fit in management field research. Academy of Management Review, 32(4), 1246-1264.

[40] Atkins, D., Eccles, M., Flottorp, S., Guyatt, G. H., Henry, D., Hill, S. et al. (2004). Systems for grading the quality of evidence and the strength of recommendations i: critical appraisal of existing approaches the grade working group. BMC Health Services Research.

[41] kitchenham, B. (2007). Guidelines for performing systematic literature reviews in software engineering. Technical report, EBSE Technical Report EBSE-2007-01.

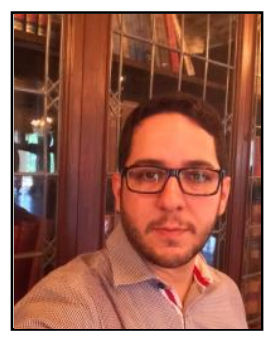

Marcelo Luiz Monteiro Marinho is currently an adjunct professor at the Department of Informatics (DEINFO) and professor at Postgraduate Programme in administration and rural development (PADR) at Federal Rural University of Pernambuco in Brazil (UFRPE). Ph.D. in computing science (2015) at Informatics Centre at Federal University of Pernambuco (CIn-UFPE) in software project management area. He holds a masters in computer science (2010) at University Federal of Pernambuco (UFPE) and graduated in computer science at Catholic University of Pernambuco. He has been working in the Software field for 11 years in which 6 with software project management. Besides, he has interest in the following lines of research are software project management, uncertainty management in software project, 
project management in global software development, global software development, innovation in software projects, software development process, and agile project.

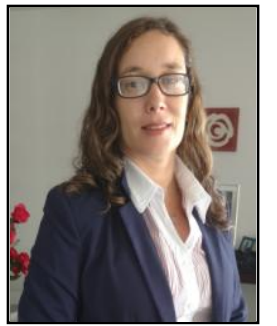

Suzana Cândido de Barros Sampaio has an extensive experience on the software development industry (19+ years) with strong expertise as consultant (10 years) in process improvement, agile methodology, and project management helping 40+ companies to achieve their business goals. Works as a Professor at Department of Informatics (DEINFO) at Federal Rural University of Pernambuco in Brazil (UFRPE), since 2013. She holds a doctoral degree (2015) and a master's degree (2010) both in computer science from Federal University of Pernambuco (UFPE). She earned a bachelor of computer science in 2000 from Catholic University of Pernambuco (UNICAP). She is also a lead auditor (ISO 9001:2015, ISO 29110), an implementer in several models such as CMMI-DEV, CMMI-SVC, MR-MPS-SV and MR-MPS-SW, to name a few. Besides, she has interest in the following lines of research: project management; agile project management; project innovation; and adaptive management.

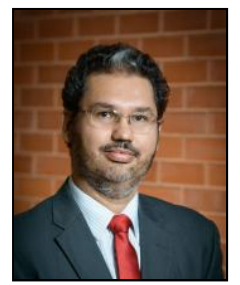

Alexandre José Henrique de Oliveira Luna is $\mathrm{CIO}$ and researcher at Clinics Hospital of the Federal University of Pernambuco (UFPE), as well researcher at Computer Centre (CIn-UFPE). He holds Ph.D. in computer science at the CIn-UFPE, Brazil; with collaborative (sandwich) exchange period at the University of British Columbia (UBC), Canada. He has an over 20-year career as scholar and practitioner in software engineering, IT Management, and Computer Science, from which last nine years have been dedicated to developing practical knowledge on agile governance topic. He holds professional certification on ITIL, COBIT, CSM and he is a PMI member. His main research interests include are agile governance, information systems, agile methodologies, software engineering, project management, and mangve.

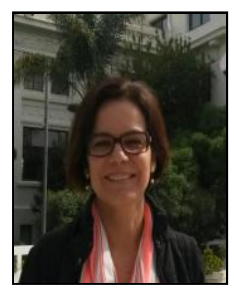

Telma Lúcia de Andrade Lima is a lecturer at the Federal Rural University of Pernambuco (UFRPE), Brazil. She holds a doctorate in production engineering from Federal University of Pernambuco (UFPE), a master in production engineering from UFPE and bachelor in mathematics from UFRPE, Brazil. She is currently acting as lecturer, teaching decision support systems and knowledge management, and as researcher. Her research interests include negotiation, partnerships, innovation in new technologies, entrepreneurship, startups and knowledge management

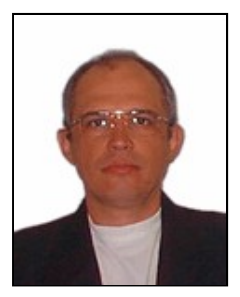

Hermano Perrelli de Moura is an associate professor and prorector for planning and finance, Federal University of Pernambuco (Recife, Brazil). PhD in computing science (1993), University of Glasgow, Scotland. MSc in computing science (1989), Federal University of Pernambuco, Brazil. A electronic engineer degree (1982) from Federal University of Pernambuco, Brazil. PMP - project management professional (2003) by Project Management Institute. Research areas of interest includes: management of software projects; software process improvement; strategic planning of information systems. Acted as a consultant on various projects involving definition and implementation of processes for project management and software development. 